\title{
Letter to the Editor: SFlt-1 and PIGF Levels in Pregnancies Complicated by SARS-CoV-2 Infection
}

\author{
Valentina Giardini $\left.{ }^{1, *} \mathbb{(}\right)$, Sara Ornaghi ${ }^{1} \mathbb{D}$, Eleonora Acampora ${ }^{1}$, Maria Viola Vasarri ${ }^{1}{ }^{\mathbb{D}}$, Francesca Arienti ${ }^{1}$, \\ Carlo Gambacorti-Passerini ${ }^{2}$, Marco Casati ${ }^{3}$ D, Andrea Carrer ${ }^{2}$ and Patrizia Vergani $^{1}$ \\ 1 Department of Obstetrics and Gynecology, MBBM Foundation, San Gerardo Hospital, University of \\ Milano-Bicocca, 20900 Monza, Italy; sara.ornaghi@gmail.com (S.O.); e.acampora@campus.unimib.it (E.A.); \\ m.vasarri@campus.unimib.it (M.V.V.); f.arienti9@campus.unimib.it (F.A.); patrizia.vergani@unimib.it (P.V.) \\ 2 Hematology Division, ASST-Monza, San Gerardo Hospital, University of Milano-Bicocca, 20900 Monza, Italy; \\ carlo.gambacorti@unimib.it (C.G.-P.); mail.carrer@gmail.com (A.C.) \\ 3 Laboratory Medicine, ASST-Monza, San Gerardo Hospital, University of Milano-Bicocca, 20900 Monza, Italy; \\ m.casati@asst-monza.it \\ * Correspondence: valentinagiardini1985@gmail.com
}

Keywords: COVID-19; SARS-CoV-2; angiogenic factors; sFlt-1; endothelial dysfunction

\section{check for}

updates

Citation: Giardini, V.; Ornaghi, S.; Acampora, E.; Vasarri, M.V.; Arienti, F.; Gambacorti-Passerini, C.; Casati, M.; Carrer, A.; Vergani, P. Letter to the Editor: SFlt-1 and PlGF Levels in Pregnancies Complicated by SARS-CoV-2 Infection. Viruses 2021, 13, 2377. https://doi.org/10.3390/ v13122377

Academic Editors: David Baud and Leó Pomar

Received: 16 November 2021 Accepted: 22 November 2021 Published: 27 November 2021

Publisher's Note: MDPI stays neutral with regard to jurisdictional claims in published maps and institutional affiliations.

Copyright: (c) 2021 by the authors. Licensee MDPI, Basel, Switzerland. This article is an open access article distributed under the terms and conditions of the Creative Commons Attribution (CC BY) license (https:/ / creativecommons.org/licenses/by/ $4.0 /)$.

\section{Dear Editor}

We read with interest the work by Espino-y-Sosa and colleagues [1], who recently reported that the Soluble Fms-like Tyrosine Kinase-1/ Angiotensin-II (sFlt-1/ANG-II) ratio could be a good predictor of adverse outcomes, including pneumonia, intensive care unit (ICU) admission, intubation, viral sepsis, and death, among pregnant women with COVID-19.

COVID-19 is a respiratory infection characterized by signs and symptoms associated with the dysfunction of the renin-angiotensin system (RAS). RAS activation leads to the formation of ANG II, a powerful vasoconstrictor and promotor of inflammation, fibrosis, and coagulation [2]; it also induces the release of sFlt-1 in case of hypoxia [3]. sFlt-1 is the soluble receptor of placental growth factor (PlGF), a potent angiogenic factor, and it antagonizes PlGF's activity in the circulation, thus creating an anti-angiogenic state and ensuing endothelial dysfunction (ED) [4].

SARS-CoV-2 invades the respiratory mucosa of the host via the Angiotensin-Converting Enzyme 2 (ACE2) receptor. ACE2 is an important element of RAS [5], and catalyzes ANG II into angiotensin 1-7 (ANG 1-7), a vasodilator with the opposite function of ANG II. Use of the ACE2 receptor for viral entry leads to the downregulation of its synthesis, with the subsequent amplification of ANG II actions and, in turn, vasospasm, inflammation, microvascular thrombosis, and organ damage. In line with these observations, a linear association between serum ANG II levels and viral load and lung damage in COVID-19 patients has been reported [6].

Preeclampsia (PE), a pregnancy-specific hypertensive disorder with multisystem involvement, is characterized by increased sensitivity to ANG II and ED, with high sFlt-1 and low PlGF values. Currently, the sFlt1/PlGF ratio is used as a clinical biomarker for the early detection and prognosis of PE [7].

Considering the common RAS-mediated underlying etiopathogenesis, we initially investigated the sFlt1/PlGF ratio in non-pregnant patients with COVID-19 pneumonia. We originally identified the presence of an angiogenic imbalance in COVID-19 patients, similar to that identified in PE women [8]. Precisely, levels of sFlt-1 were significantly higher in COVID-19-related pneumonia cases compared to those with pneumonia due to other causes and to healthy controls. PlGF values were not significantly affected by COVID-19, but the sFlt1/PlGF ratio was substantially higher in COVID-19-positive compared with COVID-19-negative pneumonia. Subsequently, other authors confirmed the increased 
sFlt-1 values in severe COVID-19 and identified sFlt-1 as a biomarker to predict survival and thrombotic accidents in COVID-19 patients [9].

The role of angiogenic markers in pregnancies complicated by SARS-CoV-2 infection is still unclear, possibly because of the interference of the placenta, the major extrarenal RAS site during pregnancy [10].

We then proceeded to assess the sFlt1/PlGF ratio in pregnant women with SARS$\mathrm{CoV}-2$ infection. Assessment of this ratio could be helpful in guiding the management of these patients and improving the understanding of the pathophysiology of SARS-CoV-2 infection in pregnancy. In fact, an increased incidence of PE among COVID-19 mothers has been reported, although such association is still incompletely elucidated [11,12]. In addition, a study showed that a PE-like syndrome can be induced by severe COVID-19 during pregnancy [13].

Precisely, we conducted a retrospective analysis of positive SARS-CoV-2 pregnant women admitted to our center from April 2020 to October 2021. SARS-CoV-2 infection was diagnosed by RT-PCR assay on nasopharyngeal swabs. Serum dosage of sFlt- 1 and PlGF (Cobas e801 analyzer Roche platform, Roche Diagnostics) was performed at the time of diagnosis of SARS-CoV-2 infection, before the beginning of any therapy. Patients already on therapy (enoxaparin sodium, steroids, or hydroxychloroquine) as well as patients without a chest X-ray performed at the time of hospital admission were excluded. The study population included 57 pregnant women, who were divided into two groups (Table 1): women with signs and symptoms of COVID-19 at hospital presentation $(n=20,35 \%)$ and asymptomatic women $(n=37,65 \%)$. sFlt-1/PlGF ratio was stratified using cut-off values clinically utilized for PE prediction (low risk $<38$, high risk $>85$ if before 34 weeks ${ }^{\prime}$ gestation or $>110$ if after 34 weeks' gestation) [14].

Asymptomatic women were identified at a surveillance swab required before hospital admission for obstetric reasons in $86 \%$ of cases $(n=32)$. The mean gestational age at diagnosis of SARS-CoV-2 infection was $37^{5 / 7}$ weeks in asymptomatic women and 32/7 weeks in symptomatic women $(p=0.089)$. Of note, $4(11 \%)$ asymptomatic women had radiological evidence of pneumonia compared to $16(80 \%)$ in the symptomatic group. In Espino-y-Sosa's work [1], none of the pregnant women with non-severe COVID-19 had pneumonia; it is not clear whether patients underwent radiological examination upon hospital admission.

Among our 20 COVID-19 symptomatic cases, 7 (35\%) required high-dependency/intensive care with 3 of them undergoing endotracheal intubation. Continuous positive airway pressure was applied in five cases $(25 \%)$. Eight $(40 \%)$ women delivered by cesarean section but only in two cases $(10 \%)$ for respiratory failure. The mean gestational age at delivery was $38^{2 / 7}$ weeks with a mean latency time between symptom onset and delivery of $48 \pm 44$ days. All women received therapy with enoxaparin sodium, $14(70 \%)$ were given steroids, and $2(10 \%)$ hydroxychloroquine (first pandemic wave). Of note, there were no stillbirths, or maternal or neonatal deaths, among these symptomatic patients; additionally, no cases of PE, fetal growth restriction, or small for gestational age neonates were diagnosed. Espino-y-Sosa et al. reported worse maternal and neonatal outcomes compared with our results.

Additionally, differently from Espino-y-Sosa et al., we identified higher sFlt-1 levels in asymptomatic patients compared to symptomatic patients ( $4899 \pm 4357 \mathrm{vs} .3187 \pm 2426 \mathrm{pg} / \mathrm{mL}$, $p=0.005$ ). sFlt- $1 / \mathrm{PlGF}$ ratio at admission was $\leq 38$ in 18 of the 20 symptomatic women compared to $22(59 \%)$ of the asymptomatic patients (mean gestational age at admission $32^{1 / 7}$ weeks versus $38^{3 / 7}$ weeks) $(p=0.018)$. In turn, rates of patients with sFlt-1/PlGF ratio at admission $>85 / 110$ were similar between symptomatic and asymptomatic group ( $n=0$ versus $n=4,11 \% ; p=0.286$ ).

This difference in the increase in sFlt- 1 between our data and those reported by Espino$\mathrm{y}$-Sosa and colleagues may be due to the higher gestational age at hospital admission of our asymptomatic patients. An additional explanation might be the delayed hospital access of Espino-y-Sosa's severe COVID-19 patients, leading to increased sFlt-1 levels due 
to prolonged exposure to hypoxia [3]. In our study, the mean number of days between symptom onset and hospital admission was five. Furthermore, we performed the assay of sFlt-1 and PIGF on serum according to the instructions of Roche Diagnostics; in Espinoy-Sosa's work, it is unclear whether sFlt-1 and PlGF tests were performed on plasma or serum and whether these women received pre-hospital treatment that may have affected the markers analyzed.

No significant differences in the incidence of PE were identified between severe and non-severe COVID-19 patients in Espino-y-Sosa's work, as well as in our cohort. A recent sub-analysis from the INTERCOVID study population showed that COVID-19 during pregnancy is independently associated with PE. Interestingly, this association is not modified by COVID-19 severity [15]. sFlt-1/PIGF ratio results among SARS-CoV-2infected asymptomatic women are of particular interest. If the increase in sFlt- 1 levels we observed is the consequence of viral infection, and not just of the higher gestational age at its evaluation, then the identification of asymptomatically infected pregnant women might be important since they may benefit from more intensive antenatal surveillance of fetal growth and blood pressure due to a potential increased risk of developing PE. Furthermore, the data from this study provide further reason to push pregnant women to be vaccinated, given the possible obstetric complications in the case of asymptomatic SARS-CoV-2 infection. In conclusion, our data suggest that SARS-CoV-2 infection during pregnancy could influence the angiogenic profile, but likely with a different effect according to the type of viral infection (symptomatic versus asymptomatic). Further research in a larger prospective cohort is needed.

Table 1. Population characteristics comparing positive SARS-CoV-2 pregnant women with signs and symptoms of COVID19 at admission to asymptomatic women.

\begin{tabular}{|c|c|c|c|c|}
\hline SARS-CoV-2 Pregnant Women & All & Asymptomatic & Symptomatic & \multirow{2}{*}{$p$ Value } \\
\hline Variables & $n=57$ & $37(65)$ & $20(35)$ & \\
\hline \multicolumn{5}{|l|}{ Anamnestic Characteristics } \\
\hline Age (years) & $33 \pm 5$ & $33 \pm 5$ & $33 \pm 4$ & 0.667 \\
\hline Italian & $30(53)$ & $20(54)$ & $10(50)$ & 0.788 \\
\hline Nulliparous & $23(40)$ & $14(38)$ & $6(30)$ & 0.772 \\
\hline Obesity (BMI > 30 kg/m²) & $12(21)$ & $7(19)$ & $5(25)$ & 0.736 \\
\hline Diabetes/Gestational diabetes mellitus & $15(26)$ & $10(27)$ & $5(25)$ & 0.580 \\
\hline Chronic hypertension & $2(4)$ & $2(5)$ & 0 & 0.536 \\
\hline \multicolumn{5}{|l|}{ SARS-CoV-2 infection } \\
\hline GA at positive swab (weeks.days \pm weeks) & $35.5 \pm 6$ & $37.5 \pm 5$ & $32 \pm 6$ & 0.089 \\
\hline Respiratory symptoms at admission & $16(28)$ & 0 & $16(80)$ & 0.0001 \\
\hline Pneumonia on chest $\mathrm{x}$-ray & $20(35)$ & $4(11)$ & $16(80)$ & 0.0001 \\
\hline High dependency unit admission & $6(11)$ & 0 & $6(30)$ & 0.001 \\
\hline ICU admission & $3(5)$ & 0 & $3(15)$ & 0.039 \\
\hline Oxygen supplementation & $14(25)$ & 0 & $14(70)$ & 0.0001 \\
\hline Continuous positive airway pressure (CPAP) & $5(9)$ & 0 & $5(25)$ & 0.004 \\
\hline Intubation & $3(5)$ & 0 & $3(15)$ & 0.039 \\
\hline Maternal/fetal/neonatal death & 0 & 0 & 0 & NA \\
\hline
\end{tabular}


Table 1. Cont.

\begin{tabular}{|c|c|c|c|c|}
\hline SARS-CoV-2 Pregnant Women & All & Asymptomatic & Symptomatic & Valu \\
\hline Variables & $n=57$ & 37 (65) & $20(35)$ & $p$ value \\
\hline \multicolumn{5}{|l|}{ Angiogenic factors } \\
\hline GA at blood test (weeks.days \pm weeks) & $36.2 \pm 6$ & $38.3 \pm 4$ & $32.1 \pm 6$ & 0.208 \\
\hline Latency timeCOVID-19 symptoms_-blood test (days) & $14 \pm 24$ & $27 \pm 34$ & $5 \pm 4$ & 0.038 \\
\hline sFlt-1 (pg/mL) & $4948 \pm 3988$ & $4899 \pm 4357$ & $3187 \pm 2426$ & 0.005 \\
\hline PlGF (pg/mL) & $237 \pm 178$ & $178 \pm 104$ & $346 \pm 232$ & 0.099 \\
\hline sFlt1/PlGF ratio & $38 \pm 50$ & $50 \pm 58$ & $17 \pm 23$ & 0.099 \\
\hline sFlt1/PlGF $<38$ & $40(70)$ & $22(59)$ & $18(90)$ & 0.018 \\
\hline sFlt1/PlGF > 85/110 (if before/after 34 weeks) & $4(7)$ & $4(11)$ & 0 & 0.286 \\
\hline \multicolumn{5}{|l|}{ Pregnancy } \\
\hline Hypertensive disorders of pregnancy/post-partum & $5(9)$ & $4(11)$ & $1(5)$ & 0.647 \\
\hline Fetal growth restriction & $3(5)$ & $3(8)$ & 0 & 0.545 \\
\hline Premature birth $<37$ weeks & $4(7)$ & 0 & $4(20)$ & 0.012 \\
\hline Small for gestational age newborn & $6(11)$ & $6(16)$ & 0 & 0.081 \\
\hline
\end{tabular}

Data presented as mean \pm standard deviation or $n(\%)$. GA: gestational age. Fetal growth restriction: Delphi consensus methodology [16]. Small for gestational age newborn: birthweight below the 10th percentile (INeS charts) [17].

Author Contributions: Conceptualization, V.G.; methodology, V.G.; formal analysis, V.G.; writingoriginal draft preparation, V.G.; writing-review and editing, S.O., P.V., C.G.-P., M.C. and A.C.; supervision, P.V.; data collection, V.G., E.A., M.V.V. and F.A. All authors have read and agreed to the published version of the manuscript.

Funding: This research received no external funding.

Institutional Review Board Statement: The study was conducted according to the guidelines of the Declaration of Helsinki, and approved by the Ethics Committee of MBBM Foundation (AMICO study-16 July 2020).

Informed Consent Statement: Informed consent was obtained from all subjects involved in the study.

Data Availability Statement: The data that support the findings of this study are available on request from the corresponding author.

Conflicts of Interest: The authors declare no conflisct of interest.

\section{References}

1. Espino-y-Sosa, S.; Martinez-Portilla, R.J.; Torres-Torres, J.; Solis-Paredes, J.M.; EstradaGutierrez, G.; Hernandez-Pacheco, J.A.; Espejel-Nuñez, A.; Mateu-Rogell, P.; Juarez-Reyes, A.; Lopez-Ceh, F.E.; et al. Novel Ratio Soluble Fms-like Tyrosine Kinase1/Angiotensin-II (sFlt-1/ANG-II) in Pregnant Women Is Associated with Critical Illness in COVID-19. Viruses 2021, $13,1906$. [CrossRef] [PubMed]

2. Benigni, A.; Cassis, P.; Remuzzi, G. Angiotensin II revisited: New roles in inflammation, immunology and aging. EMBO Mol. Med. 2010, 2, 247-257. [CrossRef] [PubMed]

3. Murphy, S.R.; Cockrell, K. Regulation of soluble fms-like tyrosine kinase-1 production in response to placental ischemia/hypoxia: Role of angiotensin II. Physiol. Rep. 2015, 3, e12310. [CrossRef] [PubMed]

4. Maynard, S.E.; Min, J.Y.; Merchan, J.; Lim, K.H.; Li, J.; Mondal, S.; Libermann, T.A.; Morgan, J.P.; Sellke, F.W.; Stillman, I.E.; et al. Excess placental soluble fms-like tyrosine kinase 1 (sFlt1) may contribute to endothelial dysfunction, hypertension, and proteinuria in preeclampsia. J. Clin. Investig. 2003, 111, 649-658. [CrossRef] [PubMed]

5. Cheng, H.; Wang, Y.; Wang, G.Q. Organ-protective effect of angiotensin-converting enzyme 2 and its effect on the prognosis of COVID-19. J. Med. Virol. 2020, 92, 726-730. [CrossRef] [PubMed]

6. Liu, Y.; Yang, Y.; Zhang, C.; Huang, F.; Wang, F.; Yuan, J.; Wang, Z.; Li, J.; Li, J.; Feng, C.; et al. Clinical and biochemical indexes from 2019-nCoV infected patients linked to viral loads and lung injury. Sci. China Life Sci. 2020, 63, 364-374. [CrossRef] [PubMed]

7. Stepan, H.; Hund, M.; Andraczek, T. Combining Biomarkers to Predict Pregnancy Complications and Redefine Preeclampsia: The Angiogenic-Placental Syndrome. Hypertension 2020, 75, 918-926. [CrossRef] [PubMed] 
8. Giardini, V.; Carrer, A.; Casati, M.; Contro, E.; Vergani, P.; Gambacorti-Passerini, C. Increased sFLT-1/PIGF ratio in COVID-19: A novel link to angiotensin II-mediated endothelial dysfunction. Am. J. Hematol. 2020, 95, E188-E191. [CrossRef] [PubMed]

9. Negro, A.; Fama, A.; Penna, D.; Belloni, L.; Zerbini, A.; Giuri, P.G. SFLT-1 levels in COVID-19 patients: Association with outcome and thrombosis. Am. J. Hematol. 2021, 96, E41-E43. [CrossRef] [PubMed]

10. Verdonk, K.; Visser, W.; Van Den Meiracker, A.H.; Danser, A.H. The renin-angiotensin-aldosterone system in pre-eclampsia: The delicate balance between good and bad. Clin. Sci. 2014, 126, 537-544. [CrossRef] [PubMed]

11. Ahlberg, M.; Neovius, M.; Saltvedt, S.; Söderling, J.; Pettersson, K.; Brandkvist, C.; Stephansson, O. Association of SARS-CoV-2 Test Status and Pregnancy Outcomes. JAMA 2020, 324, 1782-1785. [CrossRef] [PubMed]

12. Wei, S.Q.; Bilodeau-Bertrand, M.; Liu, S.; Auger, N. The impact of COVID-19 on pregnancy outcomes: A systematic review and meta-analysis. CMAJ 2021, 193, E540-E548. [CrossRef] [PubMed]

13. Mendoza, M.; Garcia-Ruiz, I.; Maiz, N.; Rodo, C.; Garcia-Manau, P.; Serrano, B.; Lopez-Martinez, R.M.; Balcells, J.; FernandezHidalgo, N.; Carreras, E.; et al. Pre-eclampsia-like syndrome induced by severe COVID-19: A prospective observational study. BJOG 2020, 127, 1374-1380. [CrossRef] [PubMed]

14. Herraiz, I.; Llurba, E.; Verlohren, S.; Galindo, A.; Spanish Group for the Study of Angiogenic Markers in Preeclampsia. Update on the Diagnosis and Prognosis of Preeclampsia with the Aid of the sFlt-1/PlGF Ratio in Singleton Pregnancies. Fetal Diagn. Ther. 2018, 43, 81-89. [CrossRef] [PubMed]

15. Papageorghiou, A.T.; Deruelle, P.; Gunier, R.B.; Rauch, S.; García-May, P.K.; Mhatre, M.; Usman, M.A.; Abd-Elsalam, S.; Etuk, S.; Simmons, L.E.; et al. Preeclampsia and COVID-19: Results from the INTERCOVID prospective longitudinal study. Am. J. Obstet. Gynecol. 2021, 225, e1-e289. [CrossRef] [PubMed]

16. Gordijn, S.J.; Beune, I.M.; Thilaganathan, B.; Papageorghiou, A.; Baschat, A.A.; Baker, P.N.; Silver, R.M.; Wynia, K.; Ganzevoort, W. Consensus definition of fetal growth restriction: A Delphi procedure. Ultrasound Obstet. Gynecol. 2016, 48, 333-339. [CrossRef] [PubMed]

17. Bertino, E.; Di Nicola, P.; Varalda, A.; Occhi, L.; Giuliani, F.; Coscia, A. Neonatal growth charts. J. Matern. Fetal Neonatal Med. 2012, 25 (Suppl. 1), 67-69. [CrossRef] [PubMed] 